\title{
Risk of subsequent primary neoplasms developing in lung cancer patients with prior malignancies
}

\author{
Malcolm V. Brock, MD \\ Anthony J. Alberg, PhD \\ Craig M. Hooker, MPH \\ Anne L. Kammer, RHIT, CTR \\ Li Xu, BS \\ Carmen M. Roig, RN \\ Stephen C. Yang, MD
}

\footnotetext{
From The Johns Hopkins Medical Institutions, Baltimore, Md.

Dr Brock is a recipient of a research supplement award from the National Cancer Institute (grant CA84986). Dr Alberg is a recipient of a K07 award (CA73790) from the National Cancer Institute. This work was also supported in part by National Institutes of Health grant CA58184.
}

Read at the Eighty-third Annual Meeting of The American Association for Thoracic Surgery, Boston, Mass, May 4-7, 2003.

Received for publication May 2, 2003; revisions requested Sept 29, 2003; revisions received Oct 6, 2003; accepted for publication Oct 22, 2003.

Address for reprints: Stephen C. Yang, MD, 624 Osler, $600 \mathrm{~N}$ Wolfe St, Baltimore, MD 21287 (E-mail: syang@jhmi.edu).

J Thorac Cardiovasc Surg 2004;127:1119-25

0022-5223/\$30.00

Copyright (C) 2004 by The American Association for Thoracic Surgery

doi:10.1016/j.jtcvs.2003.10.039
Background: This study was performed to test the hypothesis that a history of other primary neoplasms before a lung cancer diagnosis increases the risk of subsequent malignancy.

Methods: Of 8363 lung cancer patients seen from 1978 to 2002, 881 (11\%) had at least 1 previous nonlung primary malignancy. Charts were analyzed for the occurrence of subsequent malignancies.

Results: Lung cancer diagnosis in 881 patients consisted of $75 \%$ non-small cell, $12 \%$ small cell, and $13 \%$ other histologies. The median age was 66 years, with $56 \%$ male, $76 \%$ white, and $86 \%$ smokers. Of the 881 patients, $92 \%$ had no subsequent cancer (group 1), and $8 \%$ went on to experience the development of a new primary neoplasm (including lung) after their lung cancer (group 2). After adequate followup, the cumulative probability of developing a subsequent cancer did not differ markedly between those with and without a prior non-lung cancer diagnosis at 2 years $(12 \%$ vs $10 \%)$ or 5 years (16\% vs $15 \%)$. Group 1 patients had a significantly lower 1 - and 5-year survival than group 2 patients $(59 \%$ vs $48 \%$ and $29 \%$ vs $17 \%$, respectively; $P=.008$ ). Although multivariate analysis suggested that stage, history of tobacco-associated neoplasm, and history of definitive surgical resection were important determinants in predicting long-term survival, a prior malignancy was not an independent risk factor in the development of subsequent malignancy.

Conclusions: The risk of developing a subsequent malignancy is very high in lung cancer patients with prior primary malignancies, but it is not markedly different from the risks experienced by patients with no prior malignancies.

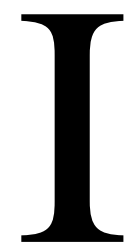

$\mathrm{n}$ the United States, lung cancer is the leading cause of cancer deaths in men and women and accounts for nearly one third of all cancer mortality. ${ }^{1}$ With the aging population and improvements in medical therapy, lung cancer patients with a history of 1 or more previous primary tumors are presenting with increasing frequency. Little is known about subsequent cancer risks and associations in these lung cancer patients with multiple independent primaries (MIPs).

In this study, our primary objective was to evaluate the risk of development of a subsequent (synchronous or metachronous) neoplasm in lung cancer patients with previous malignancies. Our hypothesis was that lung cancer patients with MIPs had an increased risk of subsequent cancers compared with lung cancer patients with no previous malignancies. The published data suggest that the risk of developing a second lung cancer in patients who survive a non-small-cell lung cancer is approximately $1 \%$ to $2 \%$ per patient per year. ${ }^{2}$ For small-cell lung cancer, it is approxi- 
TABLE 1. Distribution of subjects by prior and subsequent cancer diagnoses $(n=881)$

\begin{tabular}{lcrccr}
\hline $\begin{array}{l}\text { Number of cancer } \\
\text { diagnoses before } \\
\text { first lung cancer }\end{array}$ & \multicolumn{5}{c}{$\begin{array}{c}\text { Number of cancer diagnoses after first lung } \\
\text { cancer diagnosis }\end{array}$} \\
\cline { 2 - 6 } diagnosis & $\mathbf{0}$ & $\mathbf{1}$ & $\mathbf{2}$ & $\mathbf{3}$ & Total \\
\hline 1 & $686^{*}$ & 52 & 5 & 3 & 746 \\
2 & $107^{*}$ & 9 & 1 & 0 & 117 \\
$\geq 3$ & $16^{*}$ & 2 & 0 & 0 & 18 \\
Total & $809^{*}$ & $63 \dagger$ & $6 \dagger$ & $3 \dagger$ & 881 \\
\hline
\end{tabular}

*Group 1.

tGroup 2.

mately $6 \%$ per patient per year. ${ }^{2}$ For lung cancer patients with previous malignancies, the rate of developing subsequent aerodigestive tract tumors has not been widely reported.

\section{Patients and Methods}

From 1978 to 2002, 881 primary lung cancer patients with a history of at least 1 malignancy in a site other than the lung presented to the Johns Hopkins Hospital and made up our study population. During the same period, 8005 lung cancer patients with no history of malignancy presented to the same institution and comprised our reference population. The study group was then further subdivided into patients who had lung cancer as their last cancer diagnosis and did not experience subsequent tumors (group $1 ; \mathrm{n}=809)$ and patients who had additional neoplasms after their primary lung cancer (group $2 ; \mathrm{n}=72$ ). All malignant cases entered into the hospital's cancer registry contain specific data on past neoplasms to comply with internal requirements. Patients with previous nonmelanoma skin cancers and diagnoses made at autopsy or at death were excluded from the study population. Approval to perform this study was obtained from the Institutional Review Board of the Johns Hopkins Medical Institutions.

The number of multiple primary tumors in any case series can vary greatly according to the definitions used, and strict local rules were established to prevent misclassification of cancer metastases to the lung as primary lung tumors. We defined multiple primary tumors according to the National Cancer Database American College of Surgeons' rules to ensure consistent reproducibility between coders. Data collection guidelines for multiple primary tumors were followed by using the Facility Oncology Registry Data Standards developed by the American College of Surgeons. ${ }^{3}$ These standards define MIPs as 2 or more tumors arising at different sites (defined by the first 3 digits of the International Classification of Diseases for Oncology code) ${ }^{4}$ or at the same sites when histologic characteristics differ. In cases of second tumors in the lung, the question of whether the new lesion is a metastasis or a second primary lung tumor is not always apparent. To avoid misclassification, coders considered the criteria of Martini and Melamed $^{5}$ when classifying tumors of identical histology as multiple, independent lung primary tumors. In extreme cases, the cancer registrar manager actively solicited the assistance of the physician, the surgeon, and the pathologist for verification.
Both passive (via computer networks with national and regional death/vital statistics data, as well as other hospital-based registries) and active (telephone interviews) follow-up helped to restrict losses to follow-up to less than $1 \%$ of total cases. Vital status data were provided by the National Death Index of the National Center Health Statistics for all study subjects not lost to follow-up as of August 2002.

To compare the different patient groups, the $\chi^{2}$ test of independence was used for categorical variables, and the $t$ test was used for continuous variables. For analyses of cancer incidence, each patient's follow-up time was determined as the time from the index lung cancer diagnosis to the first subsequent tumor or death, loss to follow-up, or the end of the study period (Aug 31, 2002). For analyses of survival, each patient's follow-up time was determined as the time from the index lung cancer diagnosis to death, loss to follow-up, or the end of the study period. We classified cancer sites as tobacco associated and non-tobacco associated according to the definition of the International Agency for Research on Cancer. ${ }^{6}$ According to this definition, tobacco-associated malignancies included cancers of the oral cavity and pharynx, larynx, esophagus, lung, pancreas, stomach, bladder, kidney, liver, and cervix. Malignancies from any other site were considered non-tobacco associated.

The time from lung cancer diagnosis to subsequent cancer was modeled by using the Kaplan-Meier method and was compared by using the log-rank test for univariate analyses. Cox proportional hazards models were fit to estimate the relative risks and $95 \%$ confidence intervals associated with risks of subsequent cancer and risks of death after adjustment for covariates. Stata statistical software (Stata Corp, College Station, Tex) was used for all analyses. All reported $P$ values are 2 sided.

\section{Results}

The study population consisted of 881 patients with 1128 neoplasms: 952 prior cancers and 176 subsequent cancers. The experience of prior and subsequent cancer diagnoses is summarized in Table 1 . The vast majority (85\%) of all patients had only a single prior malignancy. This proportion did not differ between patients who did (83\%) and did not $(85 \%)$ have a subsequent malignancy. Both the prior $(30 \%$; 284/952) and subsequent (44\%; 78/176) neoplasms were heavily weighted toward the tobacco-associated malignancies-proportions that increased to $38 \%$ and $58 \%$, respectively, if only the tumors that presented immediately before and after the lung cancer primary tumor were considered.

The 2- and 5-year cumulative probability of developing a subsequent cancer diagnosis after lung cancer with a prior non-lung cancer diagnosis was $12 \%$ and $16 \%$, respectively (Table 2). In comparison, the 2- and 5-year cumulative probability of developing a subsequent cancer diagnosis after lung cancer without a prior cancer diagnosis was $10 \%$ and $15 \%$, respectively (Table 2). A prior malignancy, therefore, is not an independent risk factor for developing subsequent malignancies in lung cancer patients.

By univariate analysis, the patients in the study population who had subsequent tumors after lung cancer (group 2) 
TABLE 2. The cumulative probability of developing a subsequent malignancy after lung cancer by patients with a previous nonlung malignancy $(A ; n=881)$ and patients with no previous malignancy $(B ; n=8005)$

\begin{tabular}{|c|c|c|c|c|c|c|c|c|}
\hline \multirow{2}{*}{$\begin{array}{l}\text { Time } \\
\text { (mo) }\end{array}$} & \multicolumn{2}{|c|}{ Individuals at risk } & \multicolumn{2}{|c|}{ Number of events } & \multicolumn{2}{|c|}{$\begin{array}{c}\text { Number censored or } \\
\text { withdrawn }\end{array}$} & \multicolumn{2}{|c|}{$\begin{array}{c}\text { Cumulative } \\
\text { probability of } \\
\text { subsequent cancer }\end{array}$} \\
\hline & A & B & A & B & A & B & A & B \\
\hline 0 & 881 & 8005 & 40 & 300 & 264 & 2895 & .05 & .05 \\
\hline 6 & 577 & 4810 & 4 & 27 & 169 & 1623 & .06 & .06 \\
\hline 12 & 404 & 3160 & 7 & 29 & 103 & 885 & .08 & .07 \\
\hline 18 & 294 & 2246 & 2 & 22 & 67 & 458 & .09 & .08 \\
\hline 24 & 225 & 1766 & 7 & 26 & 55 & 494 & .12 & .10 \\
\hline 36 & 163 & 1246 & 4 & 23 & 33 & 268 & .14 & .12 \\
\hline 48 & 126 & 955 & 2 & 8 & 27 & 166 & .16 & .13 \\
\hline 60 & 97 & 781 & 0 & 13 & 16 & 109 & .16 & .15 \\
\hline
\end{tabular}

were more likely than those who did not (group 1) to be diagnosed with early-stage (stages I and II) disease of their index lung tumor $(P<.01)$ and were more likely to have had a curative operation $(P<.013$; Table 3$)$. Of the 278 patients with stage I and stage II disease, $11 \%$ went on to have subsequent cancers, compared with $6 \%$ of the 504 patients with stage III and IV cancer $(P<.01)$. The comparisons of patient demographics, smoking habits, occupational exposures, and tumor histology revealed no remarkable differences between the 2 groups. When age, sex, smoking habits, stage, surgical treatment, history of prior tobacco-associated cancers, and tumor histology were simultaneously considered in a Cox proportional hazards regression model, none of these was a significant predictor of developing a subsequent tumor.

The median time from the most recent prior cancer diagnosis to the onset of lung cancer was 58 months. The median time for tobacco-associated tumors was 53 months, versus 60 months for non-tobacco-associated malignancies (Table 4). The median time to onset from the index lung primary tumor to the next malignancy was 2.5 months (Table 5). Of 72 patients who had a subsequent primary malignancy, 44\% (32/72) had another malignancy diagnosed at the same time as their primary lung tumor. The probability of a subsequent malignancy developing synchronously or metachronously in group 2 patients was $63 \%$ $(45 / 72)$ at 12 months and 74\% (53/72) by 24 months.

By using the Cox model to analyze survival outcomes, the data suggest that a patient's stage, history of tobaccoassociated neoplasm, and history of definitive surgical resection were important determinants in predicting long-term survival (Table 6). Figure 1 demonstrates that group 2 patients had an overall survival advantage over group 1 patients for both 1- and 5-year survival (48\% vs 59\% and $17 \%$ vs $29 \%$, respectively; $P=.008$ ). In both groups, the 5 -year survival data for stage 1 patients $(45 \%$ and $48 \%$, respectively) were lower than published data for patients presenting with lung cancer alone.

\section{Discussion}

Of 8363 lung cancer patients, $881(11 \%)$ presented with a history of at least 1 prior primary malignancy at our institution in more than 2 decades. A subsequent primary cancer occurred in $8 \%$ (72/881). In this group of 72 patients, nearly $75 \%$ of patients had a new primary tumor within 2 years of their lung cancer diagnosis. Not surprisingly for a study of lung cancer patients, many of these new primary malignancies were tobacco associated.

Factors associated with the primary tumor outcome (development of subsequent malignancies) are complicated by competing variables that predict death. Patients who are current smokers, for example, would seem to be both more likely to have another malignancy as well as to have shortened survival. The 72 patients in group 2 survived longer than those with no subsequent cancer. By multivariate analysis, this prolonged survival in group 2 was associated with a higher proportion of patients with early-stage lung cancers that were amenable to curative surgical resection. A history of a tobacco-associated malignancy was a risk factor for shortened survival. By univariate analysis, tumor stage and curative resection were associated with an increased risk of subsequent malignancies. Even though the small number of patients with a subsequent tumor $(\mathrm{n}=72)$ did not allow these associations to withstand the rigors of statistical modeling, our data suggest that variables associated with prolonged survival are also risk factors for subsequent disease. This also suggests that the shared common etiologic exposures of all 881 patients with MIPs in the study place them at a very high risk for development of a subsequent malignancy. For those who had prolonged survival (group 2), the risk of another primary tumor developing within 2 years was quite high but did not differ markedly from that of lung cancer patients in general.

Few variables or measurement approaches exist that offer predictive validity to those at risk for subsequent malignancies. In this data set, patterns of organ-specific risks for subsequent neoplasms suggest common etiologic factors, 
TABLE 3. Characteristics of study population $(\mathbf{n}=\mathbf{8 8 1})$

\begin{tabular}{|c|c|c|c|c|c|}
\hline \multirow[b]{2}{*}{ Characteristic } & \multicolumn{2}{|c|}{$\begin{array}{l}\text { Group 1* } \\
(\mathrm{n}=809)\end{array}$} & \multicolumn{2}{|c|}{$\begin{array}{l}\text { Group 2† } \\
(\mathrm{n}=72)\end{array}$} & \multirow[b]{2}{*}{$P$ value } \\
\hline & No. & $\%$ & No. & $\%$ & \\
\hline \multicolumn{5}{|l|}{ Sex } & .892 \\
\hline Male & 454 & 53 & 41 & 57 & \\
\hline Female & 355 & 44 & 31 & 43 & \\
\hline Age, median (y) & 66 & - & 66 & - & .686 \\
\hline \multicolumn{5}{|l|}{ Age $(y) \ddagger$} & .884 \\
\hline$<50$ & 55 & 6.9 & 4 & 5.6 & \\
\hline $50-59$ & 139 & 17.2 & 12 & 16.7 & \\
\hline $60-69$ & 318 & 39.3 & 28 & 38.9 & \\
\hline 70-79 & 225 & 27.8 & 25 & 24.7 & \\
\hline$\geq 80$ & 72 & 8.9 & 3 & 4.2 & \\
\hline \multicolumn{5}{|l|}{ Race } & .907 \\
\hline White & 613 & 76 & 55 & 76 & \\
\hline Black/other & 196 & 24 & 17 & 24 & \\
\hline \multicolumn{5}{|l|}{ Marital status§ } & .989 \\
\hline Married & 492 & 61.2 & 44 & 61.1 & \\
\hline Not married & 312 & 38.8 & 28 & 38.9 & \\
\hline \multicolumn{5}{|l|}{ Smoking status } & .160 \\
\hline Never & 117 & 14.5 & 7 & 9.7 & \\
\hline Ever & 692 & 85.5 & 65 & 90.3 & \\
\hline Former & 315 & 38.9 & 36 & 50.0 & \\
\hline Current & 377 & 46.6 & 29 & 40.3 & \\
\hline \multicolumn{5}{|l|}{ Pack-years } & .271 \\
\hline Never & 117 & 14.5 & 7 & 9.7 & \\
\hline$<30$ & 81 & 10.0 & 6 & 8.3 & \\
\hline $30-59$ & 214 & 26.5 & 17 & 23.6 & \\
\hline $60-89$ & 140 & 17.3 & 18 & 25.0 & \\
\hline$\geq 90$ & 71 & 8.8 & 3 & 4.2 & \\
\hline Unknown & 186 & 23.0 & 21 & 29.2 & \\
\hline \multicolumn{5}{|c|}{ Hazardous occupational exposure } & .511 \\
\hline Low & 488 & 60.3 & 40 & 55.6 & \\
\hline Moderate & 112 & 13.8 & 9 & 12.5 & \\
\hline High & 101 & 12.5 & 11 & 15.3 & \\
\hline Unknown & 108 & 13.4 & 12 & 16.6 & \\
\hline \multicolumn{5}{|l|}{ Histology } & .749 \\
\hline Adenocarcinoma & 307 & 37.9 & 29 & 40.3 & \\
\hline Squamous cell & 227 & 28.1 & 22 & 30.6 & \\
\hline Large cell & 72 & 8.9 & 7 & 9.7 & \\
\hline Small cell & 98 & 12.1 & 5 & 6.9 & \\
\hline Other\| & 105 & 13 & 9 & 12.5 & \\
\hline \multicolumn{5}{|l|}{ Stage } & $.006 \emptyset$ \\
\hline 1 & 188 & 23.2 & 26 & 36.1 & \\
\hline II & 59 & 7.3 & 5 & 6.9 & \\
\hline III & 147 & 18.2 & 15 & 20.8 & \\
\hline IV & 328 & 40.5 & 14 & 19.4 & \\
\hline Unknown & 87 & 10.8 & 12 & 16.7 & \\
\hline \multicolumn{5}{|c|}{ Definitive surgical treatment received } & .013 \\
\hline Yes & 306 & 37.8 & 38 & 47.2 & \\
\hline No & 503 & 67.2 & 34 & 52.8 & \\
\hline \multicolumn{5}{|c|}{ Time from prior diagnosis closest to first lung diagnosis (y) } & .615 \\
\hline $0-1$ & 115 & 14.2 & 12 & 16.7 & \\
\hline \multirow[t]{2}{*}{$1-2$} & 107 & 13.2 & 6 & 8.3 & \\
\hline & 196 & 24.2 & 19 & 26.4 & \\
\hline $2-5$ & 196 & 24.2 & 19 & 26.4 & \\
\hline $5-10$ & 180 & 22.3 & 13 & 18.1 & \\
\hline$>10$ & 211 & 26.1 & 22 & 30.6 & \\
\hline Time to subsequent & gnos & & & & .458 \\
\hline $0-1$ & - & - & 45 & 63 & \\
\hline $1-2$ & - & - & 8 & 11 & \\
\hline
\end{tabular}

TABLE 3. continued

\begin{tabular}{|c|c|c|c|c|c|}
\hline \multirow[b]{2}{*}{ Characteristic } & \multicolumn{2}{|c|}{$\begin{array}{l}\text { Group 1* } \\
(n=809)\end{array}$} & \multicolumn{2}{|c|}{$\begin{array}{l}\text { Group 2† } \\
(\mathrm{n}=72)\end{array}$} & \multirow[b]{2}{*}{$P$ value } \\
\hline & No. & $\%$ & No. & $\%$ & \\
\hline $2-3$ & - & - & 8 & 11 & \\
\hline $3-4$ & - & - & 3 & 4 & \\
\hline $4-5$ & - & - & 2 & 3 & \\
\hline$>6$ & - & - & 6 & 8 & \\
\hline \multicolumn{5}{|c|}{ Tobacco-associated diagnoses of prior cancers } & .08 \\
\hline $\begin{array}{l}\text { Tobacco } \\
\text { associated }\end{array}$ & 266 & 32.9 & 31 & 43.1 & \\
\hline $\begin{array}{r}\text { Non-tobacco } \\
\text { associated }\end{array}$ & 543 & 67.1 & 41 & 56.9 & \\
\hline
\end{tabular}

*Lung cancer diagnosis as the last diagnosis with at least 1 prior independent cancer diagnosis.

tLung cancer diagnosis with at least 1 prior independent cancer diagnosis and with subsequent independent cancer diagnoses.

$\ddagger$ Age at first lung diagnosis.

§Married status available on only 804 patients.

|lncludes giant cell, in situ, basal cell, spindle cell, and other nonspecific histologies.

$\Uparrow P<.01 ; \chi^{2}$ test for independence.

TABLE 4. Median time for most recent prior diagnosis to lung diagnosis $(\mathbf{N}=\mathbf{8 8 1})$

\begin{tabular}{lrc}
\hline Cancers & N & Median time (mo) \\
\hline Total & 881 & 58 \\
Tobacco-associated & 332 & 53 \\
Oral cavity and pharynx & 81 & 39 \\
Larynx & 77 & 55 \\
Esophagus & 8 & 7.5 \\
Pancreas & 6 & 99.5 \\
Stomach & 11 & 35 \\
Bladder & 66 & 45.5 \\
Kidney & 24 & 46.5 \\
Liver & 1 & 67 \\
Cervix & 58 & 136 \\
& 549 & 60 \\
Non-tobacco associated & & \\
Breast & 108 & 68 \\
Prostate & 124 & 34.5 \\
Colorectal & 102 & 59.5 \\
Other diagnoses & 215 & 76
\end{tabular}

such as tobacco smoking, as critical factors in determining patient outcome. Patients stratified as the highest risks were those with a good prognosis for prolonged survival, such as a non-small-cell lung cancer patient with early-stage resectable disease and a history of a non-tobacco-associated tumor that occurred approximately 5 years previously. This same phenomenon of prolonged survival being a risk factor for subsequent disease has also been observed in population-based studies. In reviewing 141,000 Swedish women with breast cancer, Prochazka and associates ${ }^{7}$ found that those at highest risk of a subsequent lung cancer were 
TABLE 5. Median time for most recent subsequent diagnosis $(\mathbf{n}=72$ )

\begin{tabular}{lcc}
\hline Cancers & No. & Median time (mo) \\
\hline Total & 72 & 2.5 \\
Tobacco associated & 42 & 7 \\
$\quad$ Lung & 24 & 13.5 \\
Oral cavity and pharynx & 7 & 0 \\
Larynx & 2 & 0 \\
Esophagus & 2 & 0 \\
Pancreas & 2 & 11.5 \\
Stomach & 2 & 1.5 \\
Bladder & 2 & 119.5 \\
Kidney & 1 & 13 \\
Liver & - & - \\
Cervix & - & - \\
& 30 & 0.5 \\
Non-tobacco associated & & 0 \\
Breast & 6 & 8.5 \\
Prostate & 2 & 6.5 \\
Colorectal & 4 & 1.5 \\
Other diagnoses & 18 & \\
\hline
\end{tabular}

women younger than 50 years old at the time of the breast cancer diagnosis. Buiatti and associates ${ }^{8}$ found on analysis of 19,000 incident cases of the breast, colon, rectum, lung, and stomach in Italy that patients younger than 65 years old were at a considerably higher risk of the development of multiple cancers. In both of these studies, the effect of increased medical surveillance as a part of patient follow-up was speculated as a potential confounding factor.

The inclusion of information about cancers before lung cancer in a study of this size with long-term follow-up makes this study unique among investigations of subsequent primary tumors after lung cancer. Despite its size, it is still difficult in this study to discriminate precisely between those patients at most risk for experiencing subsequent malignancies and those who are not. Few studies have examined this question of MIPs in primary lung cancer patients, and most have been population-based and not hospital-based registry analyses. Although the populationbased studies have methodologic advantages compared with ours, our study provides excellent complementary evidence because of the more specific clinical data on each patient.

Liu and colleagues ${ }^{9}$ showed in 193 Taiwanese patients with MIPs involving the lung that cigarette smoking is a significant risk factor $(P<.001)$ for the development of multiple malignancies. In their study, 26\% (51/193) of lung cancer patients had lung cancer as their first malignancy and then subsequently experienced the development of a second primary tumor, whereas the remaining 142 patients had a history of malignancies antecedent to their lung cancer. In agreement with our data, they observed a significant difference in the median time of onset of previous and subsequent tumors to the index lung primary tumor (median, 46 vs 10 months, respectively, $P<.001)$. After diagnosis and treat-

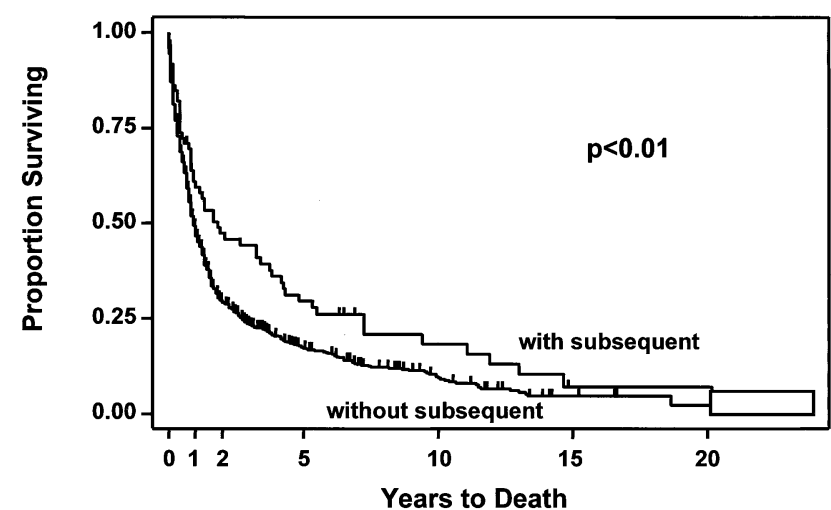

Figure 1. Survival curves of lung cancer patients with and without subsequent diagnoses.

ment of a lung tumor, we also observed a shift of risk toward neoplasms that were tobacco associated. Tobaccoassociated tumors represented only $32 \%$ of malignancies that presented immediately before the lung diagnosis but comprised $58 \%$ of subsequent tumors that presented immediately after the lung cancer primary tumor. A second primary malignancy of the lung was the most common synchronous and metachronous malignancy and occurred in $38 \%$ (27/72) of group 2 patients.

In this study, we did not limit our analysis to metachronous malignancies that occurred after the primary lung cancer diagnosis but included the risk of all subsequent tumors. This introduces the possibility that confounding factors such as overdiagnosis of occult tumors may have biased these results. This effect of increased medical surveillance can occur, for example, when a flurry of clinical examinations is performed within the first month of a lung cancer diagnosis to evaluate the behavior and extent of the lung disease. Indeed, $44 \%$ of group 2 patients had other primary tumors diagnosed synchronously with their lung primary tumor, and in the first year, 63\% had a subsequent malignancy. The iatrogenic effect after treatment for the first cancer and its effect on the incidence of any subsequent malignancy was also not considered in our analysis. The risk of lung cancer in women with previous breast cancer who have undergone radiotherapy, for example, has been shown to be significantly increased. ${ }^{7,8,10,11}$ In the future, analysis of clonal differences between distinct lesions may not only solve the current diagnostic dilemma between multiple primary cancers and metastases, but also assist in discerning the etiologic origins of MIPs from various organ sites. Both van Rens and colleagues, ${ }^{12}$ using RNA mutational analysis for $\mathrm{p} 53$, and Wang and colleagues, ${ }^{13}$ using mutational analysis for p53 and K-ras codons 12 and 13, demonstrated that molecular markers could distinguish the independent origins of multiple tumors in the lung. 
TABLE 6. Crude and adjusted risk estimates of mortality for patients with prior and subsequent diagnoses compared with patients with prior but no subsequent cancer diagnosis $(n=881)$

\begin{tabular}{|c|c|c|c|c|}
\hline \multirow[b]{2}{*}{ Characteristic } & \multicolumn{2}{|c|}{ Crude RR } & \multicolumn{2}{|c|}{ Adjusted RR* } \\
\hline & RR & $95 \%$ CI & $\mathbf{R R}$ & $95 \% \mathrm{CI}$ \\
\hline \multicolumn{5}{|l|}{ Sex } \\
\hline Female & 1.0 & Reference & 1.0 & Reference \\
\hline Male & 1.15 & $0.99-1.34$ & 1.12 & $0.95-1.31$ \\
\hline \multicolumn{5}{|l|}{ Age (y) } \\
\hline$<50$ & 1.0 & Reference & 1.0 & Reference \\
\hline $50-69$ & 0.88 & $0.66-1.29$ & 0.94 & $0.69-1.27$ \\
\hline$\geq 70$ & 0.94 & $0.69-1.28$ & 1.07 & $0.78-1.46$ \\
\hline \multicolumn{5}{|l|}{ Smoking status } \\
\hline Never & 1.0 & Reference & 1.0 & Reference \\
\hline Former & 0.92 & $0.73-1.16$ & 0.81 & $0.64-1.03$ \\
\hline Current & 1.27 & $1.01-1.58 \dagger$ & 1.06 & $0.84-1.33$ \\
\hline \multicolumn{5}{|l|}{ Histology } \\
\hline Adenocarcinoma & 1.0 & Reference & 1.0 & Reference \\
\hline Squamous cell & 1.22 & $1.01-1.46 \dagger$ & 1.06 & $0.89-1.28$ \\
\hline Large cell & 1.15 & $0.88-1.51$ & 1.04 & $0.79-1.36$ \\
\hline Small cell & 1.56 & $1.22-1.98 \ddagger$ & 0.96 & $0.75-1.23$ \\
\hline Other§ & 1.55 & $1.24-1.95 \ddagger$ & 1.14 & $0.90-1.43$ \\
\hline \multicolumn{5}{|l|}{ Disease extent } \\
\hline Stage I & 1.0 & Reference & 1.0 & Reference \\
\hline Stage II & 1.69 & $1.23-2.34 \ddagger$ & 1.82 & $1.31-2.52 \ddagger$ \\
\hline Stage III & 2.05 & $1.60-2.63 \ddagger$ & 1.57 & $1.22-2.04 \ddagger$ \\
\hline Stage IV & 4.20 & $3.40-5.20 \ddagger$ & 2.73 & 2.16-3.45ł \\
\hline Unknown & 2.26 & $1.73-2.95 \ddagger$ & 1.39 & $1.05-1.85^{*}$ \\
\hline \multicolumn{5}{|l|}{ Tobacco association } \\
\hline Non-tobacco associated & 1.0 & Reference & 1.0 & Reference \\
\hline Tobacco associated & 1.18 & $0.99-1.32$ & 1.25 & $1.09-1.50 \ddagger$ \\
\hline \multicolumn{5}{|c|}{ Definitive surgical treatment received } \\
\hline No & 3.37 & $2.86-4.00 \ddagger$ & 2.61 & $2.16-3.15 \ddagger$ \\
\hline Yes & 1.0 & Reference & 1.0 & Reference \\
\hline
\end{tabular}

${ }^{*}$ Adjusted for age, sex, smoking status, cancer histology, stage, presence of tobacco-associated tumor, and surgical treatment.

$\dagger P<.05 ; \ddagger P<.01$.

§Includes giant cell, non-small cell, in situ, basal cell, and other nonspecific histologies.

$R R$, Relative risk; $95 \% \mathrm{Cl}, 95 \%$ confidence interval.

In summary, this is one of the largest of only a few investigations to report the incidence of subsequent neoplasms in lung cancer patients with previous malignancies. There was no difference in the incidence of subsequent malignancies in lung cancer patients if there was a previous cancer. Factors associated with the development of a subsequent malignancy are also those associated with longer survival, such as early stage and curative resection. This apparent increased risk likely reflects that these patients are living longer and therefore have more opportunity for the development of a subsequent cancer. We interpret this to mean that all lung cancer patients with MIPs are at a high risk for another primary tumor, especially within 2 years. Many of these patients have received operative therapy and often have their continued medical surveillance provided exclusively by thoracic surgeons. Although there is no clear consensus for postoperative follow-up, there is a growing body of literature supporting the close monitoring of the thorax, especially every 3 to 6 months for the first 2 years, with radiologic imaging such as a chest radiograph or a computed tomographic scan. This study supports this view and underscores the importance of surgeons maintaining vigilant follow-up, especially for tobacco-associated malignancies, during the first 2 years after surgery.

We thank Kathy Bender for her technical assistance in helping to prepare this manuscript.

\section{References}

1. Schottenfeld D. Etiology and epidemiology of lung cancer. In: Harvey Pass JM, Johnson D, Turrisi A, Minna J, editors. Lung cancer. Philadelphia: Lippincott Williams \& Wilkins; 2000. p. 367.

2. Johnson BE. Second lung cancers in patients after treatment for an initial lung cancer. J Natl Cancer Inst. 1998;90:1335-45.

3. The Facility Oncology Registry Data Standards (FORDS) (2002). Chicago: Commission on Cancer, American College of Surgeons; 2002.

4. World Health Organization. International classification of diseases for oncology. 3rd ed. Geneva: World Health Organization; 2000. 
5. Martini N, Melamed MR. Multiple primary lung cancers. J Thorac Cardiovasc Surg. 1975;70:606-12.

6. IARC Monograph: Tobacco and Involuntary Smoking (June 2002). Volume 83. Lyon, France: IARC Press, International Agency for Research on Cancer; 2002.

7. Prochazka M, Granath F, Ekbom A, et al. Lung cancer risks in women with previous breast cancer. Eur J Cancer. 2002;38:1520-5.

8. Buiatti E, Crocetti E, Acciai S, et al. Incidence of second primary cancers in three Italian population-based cancer registries. Eur J Cancer. 1997;33:1829-34.

9. Liu YY, Chen YM, Yen SH, et al. Multiple primary malignancies involving lung cancer-clinical characteristics and prognosis. Lung Cancer. 2002;35:189-94.

10. Inskip PD, Boice JD Jr. Radiotherapy-induced lung cancer among women who smoke. Cancer. 1994;73:1541-3.

11. Neugut AI, Robinson E, Lee WC, et al. Lung cancer after radiation therapy for breast cancer. Cancer. 1993;71:3054-7.

12. van Rens MT, Eijken EJ, Elbers JR, et al. p53 mutation analysis for definite diagnosis of multiple primary lung carcinoma. Cancer. 2002; 94:188-96.

13. Wang X, Christiani DC, Mark EJ, et al. Carcinogen exposure, p53 alteration, and K-ras mutation in synchronous multiple primary lung carcinoma. Cancer. 1999;85:1734-9.

\section{Discussion}

Dr Joe B. Putnam, Jr (Houston, Tex). The authors present their detailed and carefully analyzed experience with lung cancer in association with a second primary malignancy. The authors appropriately expand upon the information presented by the Lung Cancer Study Group, Thomas and colleagues, in 1993, where 55 patients with long-term survival developed a cancer and 13 were nonpulmonary cancers, and also confirmed the work presented by Rice and colleagues from our institution at the recent meeting of the Society of Thoracic Surgeons.

The authors suggest that the development of subsequent malignancies is sufficiently high to warrant specific consideration by the physician. Routine post-cancer resection screening for neoplasms may take place with a simple office visit and chest radiograph or more intensive screening procedures. What is the recommendation for follow-up in these patients? There has been considerable controversy as to the value of follow-up in patients with lung cancer, specifically regarding its cost-effectiveness and ability to improve overall survival. Screening for cancer in all patients with prior malignancies would be prohibitively expensive.

In both groups, $90 \%$ to $95 \%$ were either former or current smokers. Nearly half the patients in group 1 and $40 \%$ of patients in group 2 were current smokers. Were these patients counseled and assisted to quit smoking?

I know that you plan further evaluation of the biological characteristics of these tumors, but how can you help us today? Are there any specific anatomic or histologic characteristics that would predispose patients to develop subsequent malignancies and screening for subsequent treatment?

In summary, patients who undergo curative resection of malignancies are at risk for developing subsequent malignancies. Eval- uation by a thoracic surgeon should include questions and examinations to screen for the subsequent neoplasms. Continuity of care and appropriate screening technology will be needed to optimize care and improve survival for these patients at increased risk of developing subsequent malignancy.

Dr Brock. I thank Dr Putnam very much for his comments and his analysis. Regarding the first question about the recommendations for screening, I do think that it is imperative that all of these patients be screened. Since our study looked primarily at lung cancer patients with previous malignancies, I think our data suggest that these patients are at an extremely high risk for developing new aerodigestive tract tumors. Interestingly, a quarter of all the patients who had subsequent aerodigestive tract tumors actually had a synchronous diagnosis with their lung cancer primary.

I think in follow-up, at the very least, a chest radiograph is warranted every 3 to 6 months for the first 2 years. Maybe some of these data, along with the data that were presented at the Society of Thoracic Surgeons by Dr Rice from your institution, can be the basis for future clinical trials to evaluate the role of high-resolution computed tomographic scans, or even positron emission tomography scans, in the follow-up of postoperative patients with lung cancer. If a study such as this is performed, it is imperative that surgeons take a critical and pivotal role in conducting these trials because of their crucial role in the day-to-day follow-up care of these patients.

As for your second question regarding the high degree of smoking in this population and smoking counseling, this was largely, as you know, a cancer registry study. Consequently, there was not a large-scale counseling effort to individual patients. We have, however, routinely counseled patients individually, especially after surgery. We also have launched new smoking awareness programs in the institution, but these have been rather limited in scale and generally involved only 1 or 2 medical departments. I think that as we begin to understand even more the long-term effects of cigarette smoking, having an institutional-wide program is going to be even more imperative.

As far as biological characteristics or molecular profiling of these tumors, we are actually in the process of looking at that from both a genetic and an epigenetic standpoint. I think that this is exciting, especially looking at the epigenetic possibilities, because of the translational potential of this exciting biomarker.

We did perform a multivariate analysis to see whether we could identify patterns of histologic or anatomic associations that may predispose patients to develop subsequent malignancies, and unfortunately, this proved to be disappointingly negative. Although this was a large study, only 72 patients developed subsequent tumors, and I think to answer your question adequately about the patterns of risk, we probably need to have an even larger group of patients who develop subsequent malignancies for our confidence intervals to remain narrow. 